Revue d'histoire de l'Amérique française

REVUE D.HISTOIRE DE L'AMÉRIQUE FRANÇAISE

\title{
Enseignement de la philosophie au Petit Séminaire de Québec (1765-1880)
}

Marc Lebel

Volume 18, numéro 3, décembre 1964

URI : https://id.erudit.org/iderudit/302411ar

DOI : https://doi.org/10.7202/302411ar

Aller au sommaire du numéro

Éditeur(s)

Institut d'histoire de l'Amérique française

ISSN

0035-2357 (imprimé)

1492-1383 (numérique)

Découvrir la revue

Citer ce document

Lebel, M. (1964). Enseignement de la philosophie au Petit Séminaire de Québec (1765-1880). Revue d'histoire de l'Amérique française, 18(3), 463-473.

https://doi.org/10.7202/302411ar d'utilisation que vous pouvez consulter en ligne.

https://apropos.erudit.org/fr/usagers/politique-dutilisation/ 


\section{BIBLIOGRAPHIE}

\section{ENSEIGNEMENT DE LA PHILOSOPHIE AU PETIT SÉMINAIRE DE QUÉBEC \\ $(1765-1880) *$}

\section{SOURCES}

\section{A) SOURCES MANUSCRITES}

1. Cahiers de notes d'élèves et de professeurs (ASQ). Nous donnons la liste des cahiers que nous avons cités au cours de notre travail:

M-154. 1782. (Cours de philosophie). Notes de l'abbé Charles Chauveaux, professeur. $121 \mathrm{p}$. Contient des thèses (pp. 29-34) ainsi qu'une liste des élèves qui "ont soutenu thèse en Philosophie, dans l'année 1783 au Séminaire de Québec". (p. 39). M-185. 1790. Compendium philosophiae. Notes de l'abbé Antoine Robert, professeur, $18 \mathrm{p}$.

M-175. 1790-94. (Cours de philosophie) Professeur: abbé Antoine Robert. Notes de Charles Burke. 374 p.

M-151. 1792. Philosophiae alumnie. Professeur: abbé Antoine Robert. Notes de Jos. Ol. Deguire. 146 p.

M-155. 1795. (Cours de philosophie) Professeur: abbé JeanBaptiste Castanet. Notes de Félix Gatien. 107 p.

M-172. 1796. Cursus philosophicus. Professeur: abbé Jean Raimbault. $105 \mathrm{p}$.

M-66. 1812. (Cours de philosophie) Professeur: abbé PierreFlavien Turgeon. Notes de Edward Daveluy. 149 p.

M-161. 1812. Philosophicus liber Tomus II dus. Professeur: Pierre-Flavien Turgeon. Notes de Edward Daveluy. $150 \mathrm{p}$.

M-214. 1817. (Cours de philosophie) Professeur: abbé Jérôme Demers. Notes de Toussaint Papineau. 263 p.

M-215. 1818, 1824. Institutiones Philosophicae ad usum Seminarii Quebecensis. Professeur: abbé George-Hilaire Besse- 
rer. (1824) Notes de Joseph Peticlerc (1824). Ce cahier a d'abord servi en 1818.

M-160. 1830. Institutiones philosophicae. Professeur: abbé Jérôme Demers. Notes de Edouard-Gabriel Plante. 287 p.

M-339. 1845. Compositions philosophiques. Notes de l'abbé Elzéar-Alexandre Taschereau, professeur, $161 \mathrm{p}$.

M-212. 1866. Cours de philosophie. Professeur: abbé LouisHonoré Paquet. Notes de Clovis Laflamme. $260 \mathrm{p}$.

M-992. 1876. (Cours de philosophie) Professeur: abbé Jacques Langis. Notes de Joseph Beaudoin. 168 p.

M-333. 1878. (Cours de philosophie). Notes de l'abbé OlivierElzéar Mathieu, professeur.

M-336. 1878-82. (Cours de philosophie). Notes de l'abbé Olivier-Elzéar Mathieu, professeur.

2. Thèses de philosophie (ASQ).

Nous donnons la liste des thèses que nous avons citées au cours de notre travail:

Sém. 13 No 14. 1772.

Sém. 13 No 15. 1774.

Sém. 13 No 16. 1777 ou 1778.

Sém. 13 No 17. 1797.

M-154. 1782: pp. 29-34.

Dans le recueil: Séminaire de Québec. Thèses 1775-1815, on trouvera une série de thèses soutenues en 1815 . Nous n'avons point trouvé de thèses postérieures à 1815 , bien qu'il soit certain qu'en 1859 et de nouveau en 1879 les étudiants de philosophie défendent des thèses. Le recueil précité renferme des thèses de mathématiques et de physique soutenues en $1775,1790,1792$ et 1796 .

3. Lettres, mémoires, listes de livres, factures (ASQ):

Lettre carton M, No 31: lettre de MM. Brisacier et Tiberge du Séminaire des Missions étrangères de Paris aux Messieurs du Séminaire de Québec, 19 juin 1705.

Sém. 5, No 50a: mémoire du Séminaire des Missions étrangères de Paris sur l'état présent du Séminaire de Québec présenté à Monseigneur le comte de Maurepas en 1734 .

Sém. 8, No 55a: compte de recette et de dépense fait pour le Séminaire de Québec par le Séminaire de Paris; envoyé le 20 mars 1766. 
Sém. 5, No 9b: Réponse de l'abbé Jérôme Demers à un questionnaire sur l'éducation, 5 janvier 1829.

Polygraphie 44, No 50: lettre de l'abbé François Désaulniers du Collège de Nicolet à l'abbé Jean Holmes, 6 avril 1836.

Contient la liste des ouvrages philosophiques que possède la bibliothèque du Collège de Nicolet en 1836.

Sém. 75, No 85: lettre de l'abbé Joseph Aubry à l'abbé Jean Holmes, vers juillet 1836. L'abbé Aubry, qui est alors préfet des études, recommande l'achat de certains livres.

Polygraphie 42, No 33 : lettre de l'abbé Jean Holmes à l'abbé Jérôme Demers, 30 août 1836.

Plante No 243: facture donnée à l'abbé Jean Holmes par la librairie Merlin de Paris, 13 septembre 1836.

Polygraphie 44, No 38: liste de livres vraisemblablement achetés par l'abbé Jean Holmes en Europe pour le Séminaire de Québec.

Polygraphie 8, No 32: mémoire relatif aux études préparatoires à l'état ecclésiastique présenté à Mgr Signay par les supérieurs et directeurs du Séminaire de Québec: sans date.

4. Autres sources manuscrites (ASQ)

Les archives du Séminaire possèdent plusieurs précieuses compilations faites par l'abbé Amédée Gosselin et ses successeurs. Nous avons mis à contribution:

Supérieurs, Directeurs, Officiers et professeurs du Séminaire de Québec de 1663 à 1880 . Contient des renseignements jusqu'à 1940.

Classes du Séminaire de Québec 1760-1795. Contient des listes d'élèves et quelques extraits copiés de lettres relatives à l'enseignement au Séminaire.

Séminaire de Québec. Thèses de Mathématiques, Exercices littéraires. Programmes des Etudes. Comptes rendus des journaux. Distribution des prix De 1770 à 1840. Extraits des journaux et particulièrement de la Gazette de Québec, du Canadien, du Vrai Canadien, du Courrier de Québec. Fait en Déc. 1908. A.E.G. Très riche.

Nous avons également consulté le catalogue de la bibliothèque de 1782 :

A. G. Dudevant, ptre. Catalogue des livres de la bibliothèque du Séminaire des Missions Etrangères de Québec fait dans le mois de May 1782. 
A la suite du catalogue proprement dit se trouvent des listes de livres empruntés de la bibliothèque par quelques prêtres du Séminaire qui ont vécu au XIXe siècle, dont l'abbé ElzéarAlexandre Taschereau.

Nous avons consulté le Journal de dépense du Séminaire au mois de juin 1784; on y signale l'achat d'un exemplaire de la Philosophie de Toul.

Un registre commencé en 1824 éclaire à la fois la question de l'alternance des classes, du nombre et de l'âge des écoliers: Registre destiné à conserver les listes des écoliers du Petit Séminaire de Québec. Depuis le 10 juillet 1824.

S'arrête en 1848, au moment où paraît le premier catalogue imprimé.

Nous avons dépouillé les procès-verbaux ou cahiers d'honneurs de deux académies, l'une et l'autre fondées après 1850: Académie St-Denys. Règlement. Constitution. Procès-verbaux. Vol. I (1853-1867) ; II (1868-1876) ; III (1876-1883).

Procès-verbaux de la Société Laval. Vol. I (1851-1865) ; II (1865-1870) ; III (1870-1877) ; IV (1877-1882).

Le Plumitif $d u$ Séminaire, tel que reproduit par le fichier des archives, nous a servi aux dates suivantes: 25 mars 1863; 14 janvier $1878 ; 31$ mars $1879 ; 9$ septembre $1879 ; 19$ janvier $1880 ; 22$ juin 1884 .

Nous avons également utilisé les pièces suivantes :

Fonds Viger-Verreau, carton 16, No 10 : liste des écoliers qui fréquentent les diverses classes qu'on fait au Séminaire en octobre 1790; indique l'âge et la condition des écoliers (pensionnaires ou externes).

Sém. 13,, No 32: Plaidoyers, Fables, Plan d'Education, Avertissements (1811-1830).

Sém. 75, No 15d: Les principes de l'éducation; discours prononcé par Jean Langevin à l'ouverture des Exercices littéraires et philosophiques, 8 août 1837. Nous avons consulté une copie récente.

Sém. 85, No 10a: Catalogue des livres de la bibliothèque des écoliers du Petit Séminaire, vers 1838.

Sém. 73, No 6: Liste des élèves à qui M. E. A. Taschereau a enseigné la philosophie (1842-1851; 1853-54; 1867-68). L'abbé Taschereau donne l'âge de ses élèves pour la période 1842-1851 et fait de brèves remarques sur la matière vue chaque année. Nous avons aussi consulté de l'abbé Tasche- 
reau son exemplaire des Institutiones philosophicae de M. Demers. Les marges fourmillent de notes et de références manuscrites. Voir M-157.

Université 99, No 47a: rapport du comité de philosophie au congrès de l'enseignement secondaire tenu à Québec en juin 1880 .

1. Manuels

\section{B) SOURCES IMPRIMÉES}

La bibliothèque de l'Université Laval possède une riche collection de manuels de philosophie utilisés par le Petit Séminaire de Québec. Nous énumérons ci-après les manuels que nous avons cités au cours de notre travail:

(Abbés Gigot et Camier). Institutiones philosophicae ad usum Seminarii Tullensis. Toul, 1769 (2e éd.) XXXIV-418 p.; 428 p.; 573 p.; 452 p. -12 planches; 479 p. -8 planches. $16 \mathrm{~cm}$.

Abbé Hauchecorne. Abrégé latin de philosophie avec une introduction et des notes françaises. A Paris, chez l'auteur, 1784. LII-289 p.; 279 p. -8 planches. $17 \mathrm{~cm}$.

(Anonyme). Cursus elementarius philosophiae ad usum collegiarum, continens logicam, metaphysicam, ethicam et religionis probationes. Paris, Lyon, 1823. 296 p. $17 \mathrm{~cm}$.

Abbé Jean-Baptiste Bouvier. Institutiones philosophicorum ad usum seminariorum et collegiarum. Paris, Lyon, 1824. VIII281 p. ; 289 p.; 327 p. ; $17.5 \mathrm{~cm}$.

(Abbé Jérôme Demers) Institutiones philosophicae ad usum studiosae juventutis. Quebeci, Ex typis Tho. Cary \& Socii, 1835.395 p. $22 \mathrm{~cm}$.

M. Laurentie, Introduction à la philosophie ou traité de l'origine et de la certitude des connaissances humaines. Paris, Méquignon Junior, 1829 (2e éd.). XII-510 p. $20.5 \mathrm{~cm}$.

Deux manuels du P. Tongiorgi semblent avoir servi au Séminaire. Pour les distinguer l'un de l'autre, maîtres et élèves parlent communément du grand et du petit Tongiorgi, le second manuel étant un compendium:

Institutiones philosophicae Salvatoris Tongiorgi e societate jesu philosophiae professoris in collegio romano eiusdem societatis. Romae, Ex officina soc. aurelianae, 1861-62. 415, 348,452 p. $20.5 \mathrm{~cm}$.

Institutiones philosophicae Salvatoris Tongiorgi $E$ societate jesu $a b$ eodem in compendium redactae. Romae, Typis Bernardi Morini, 1862.317 p. $21.5 \mathrm{~cm}$. 
Institutiones philosophicae moralis Salvatoris Tongiorgi $E$ societate jesu ab eodem in compendium redactae. Romae, Typis Bernardi Morini, 1863. 176 p. $21.5 \mathrm{~cm}$.

2. Prospectus, catalogues et annuaires

En 1830, le Séminaire publie un prospectus intitulé Exercices publics de philosophie. De 1831 à 1842 paraissent à peu près chaque année des prospectus qui intéressent les classes de lettres et de philosophie. Ce sont les Exercices philosophiques et littéraires. Ils nous renseignent sur le programme de la séance publique de fin d'année ainsi que sur le programme des études. En 1838-9, 1839-40, 1840-1 et 1841-2, le Séminaire publie également un Programme abrégé du cours d'études du Petit Séminaire pour l'année (...).

De 1847-48 à 1855-56, les presses de l'Abeille impriment neuf Catalogues des officiers et des élèves du Séminaire de Québec. On y trouve la liste des professeurs et des élèves, le programme des études, des renseignements sur les associations, des extraits du règlement des pensionnaires et des externes, des notes sur le Séminaire, etc. De 21 à 43 pages.

A partir de 1856-57, nous disposons des Annuaires de l'Université Laval. Ils renferment chaque année une section consacrée au Séminaire qui s'intitule: Officiers, professeurs et élèves du Séminaire de Québec pour l'année (...).

Les Annuaires de l'Université nous renseignent aussi sur les questions du baccalauréat et sur la Faculté des Arts dont les élèves de philosophie du Séminaire suivent les cours depuis 1859.

Enfin, nous avons eu la bonne fortune de retrouver le prospectus lancé par la maison Cary à la veille de l'impression du manuel de philosophie de l'abbé Jérôme Demers:

Prospectus, Quebec, 12e janvier 1835. Le recueil: Exercices publics - Séminaire de Québec 1830-42, contient un exemplaire de ce prospectus; le Canadien du 16 février 1835 le reproduit intégralement.

\section{Journaux}

Nous avons utilisé la précieuse compilation de l'abbé Amédée Gosselin qui reproduit les comptes rendus de séances publiques publiés par le Canadien.

Nous avons également cité:

Le Canadien, 12 mars 1834, 12 janvier 1835. 
Appendice des journaux de la Chambre d'Assemblée de la province du Bas-Canada. 1836. Voir l'appendice (0.0.) pour la déposition de l'abbé Jérôme Demers devant le comité chargé d'enquêter sur la question des écoles normales.

Le Journal de l'Instruction publique, XVII (juillet-août 1873), 122.

C'est cependant L'Abeille, journal hebdomadaire des écoliers du Séminaire, qui nous a le plus servi. Il paraît de 1848 à 1854, de 1858 à 1862 et enfin, de 1877 à 1881.

4. Autres sources imprimées

Acta et decreta sexti concilii provinciae quebecensis in quebecensi civitate anno domini MDCCCLXXVIII celebrati a sancta sede revisa et recognita. Quebeci, apud P.-G. Delisle, typographum, 1882.84 p. $22 \mathrm{~cm}$. Voir pp. $42 \mathrm{s.::Decretum}$ VIII, De studiis philosophicis.

Aubert de Gaspé, Philippe. Mémoires. Ottawa, G.-E. Desbarats, Imprimeur-Editeur, 1866.563 p. $22.5 \mathrm{~cm}$.

Bibliothèque. Petit Séminaire de Québec. Règlement et catalogue, 1881. Québec, Typographie de P.-G. Delisle, 1881. 59 p. $19 \mathrm{~cm}$.

Colbert de Maulevrier, Edouard Charles Victurnien, comte de Maulevrier. Voyages dans l'intérieur des Etats-Unis et au Canada, avec une introduction et des notes par Gilbert Chinard. Baltimore, The John Hopkins University Press, 1935. XXIII-87 p. $26.5 \mathrm{~cm}$. Index.

Encyclopédie ou dictionnaire raisonné des sciences, des arts et des métiers (...) 36 tomes - 3 volumes de planches. Genève, Pellet, édition commencée en 1777 . Nous avons utilisé les articles: Collège (VIII: 494-500) et Scholastiques (XXX: 274-287).

Gosselin, abbé David. Les étapes d'une classe au Petit Séminaire de Québec 1859-1868. Québec, H. Chassé, 1908. 291-11 p. $20 \mathrm{~cm}$.

Léon XIII. Encyclique Aaterni Patris. Nous avons consulté l'édition suivante:

Actes de Léon XIII : Encyclique, Motu proprio, Brefs, Allocutions, Actes de Dicastères, etc ... Paris, Maison de la Bonne Presse, s.d. Vol. I: $1878-1884.288$ p. $20 \mathrm{~cm}$.

Mandements, lettres pastorales et circulaires des évêques de Québec publiés par Mgr H. Têtu et l'abbé C.-D. Gagnon. Québec, Imprimerie générale A. Coté et Cie, 1890. Vol. VI: 18771887. 826 p. $22 \mathrm{~cm}$. 
Revue de l'Université Laval. Documents pour servir à l'histoire du Séminaire de Québec. Nous avons utilisé les documents CXV:

Plan d'éducation du Séminaire de Québec, octobre 1790 (RUL, novembre 1858: 270-274) ; CXXVI:

Plan d'éducation du Séminaire de Québec 1816 (RUL, janvier 1959 : 463-465) ; CXXIX :

Examen public du Petit Séminaire de Québec 1833 (RUL, janvier 1959: 469-473; CXXXV :

Mémoire pour accompagner la Pétition du Séminaire au Parlement Provincial - octobre 1843 (RUL, mai 1959 : 854-859). Renan, Ernest. Souvenirs d'enfance et de jeunesse. Nous avons consulté la Revue des Deux Mondes dans laquelle Renan publia en premier lieu ses Souvenirs: 1 (15 mars 1876: 241262) ; II (1er décembre 1876: 481-507) ; III (1er novembre 1880: 68-94); IV (15 décembre 1880: 721-746); V (1er novembre 1882: 5 -26); VI (15 novembre 1882: 241-261).

Rollin, Charles. Traité des Etudes ou de la manière d'enseigner et d'étudier les Belles-Lettres. Paris, Chamerot, 1845. VIII - 879 p. $27.5 \mathrm{~cm}$.

Roy, J.-Edmond. Souvenirs d'une classe au Séminaire de Québec (1867-1877). Lévis, Imprimerie de l'auteur, 1905. 529 p. $22 \mathrm{~cm}$.

Sixième centenaire de saint Thomas d'Aquin à Saint-Hyacinthe et à Québec. Québec, Typographie d'Augustin Coté et Cie, 1874.64 p. $21 \mathrm{~cm}$.

\section{DICTIONNAIRE}

Dictionnaire des lettres françaises, publié sous le direction du Cardinal Georges Grente (...) : le dix-huitième siècle. 2 vol. (A-K ; L-Z). Paris, Fayard, 1960. 607, 670 p. $27 \mathrm{~cm}$. Nous avons consulté l'article de René Poirier sur le cartésianisme au XVIIIe siècle: A-K: 268-273.

\section{III. ÉTUDES}

Ariès, Philippe. L'enfant et la vie familiale sous l'Ancien Régime. Paris, Plon, 1960. 503 p. $20.5 \mathrm{~cm}$.

Bastien, Hermas. L'enseignement de la philosophie. 2 vol. Vol. I: Au Canada français. Montréal, Editions Albert Levesque, 1936. $222 \mathrm{p} .19 \mathrm{~cm}$. Appendices. Index.

Chevallier, Jean-Jacques. Les grandes cuvres politiques de Machiavel à nos jours. Paris, Colin, 1957 (5e éd.) 406 p. 22 cm. Bibliographie. 
Dionne, N.-E. Vie de C.-F. Painchaud, prêtre, curé, fondateur du Collège de Sainte-Anne-de-la-Pocatière. Québec, Brousseau, 1894.440 p. $22 \mathrm{~cm}$.

Dubois, Emile. Le Petit Séminaire de Sainte-Thérèse (18251925). Montréal, les Editions du "Devoir", 1925. 399 p. $23 \mathrm{~cm}$.

Essais et bilans. Journées thomistes. I. Ottawa, Collège dominicain, 1935. 244 p. $23 \mathrm{~cm}$. Voir abbé Maurice Roy, Pour l'histoire du thomisme au Canada: 17-28.

Foucher, Louis. La philosophie catholique en France au XIXe siècle avant la renaissance thomiste et dans son rapport avec elle (1800-1880). Paris, Vrin, 1955. 280 p. $25 \mathrm{~cm}$. Index.

Gosselin, Abbé Amédée. L'instruction au Canada sous le Régime français (1635-1760). Québec, Laflamme et Proulx, 1911. 501 p. $23 \mathrm{~cm}$.

Groulx, Abbé Lionel. L'enseignement français au Canada. 2 vol. Vol I: Dans le Québec. Montréal, Librairie d'action canadienne-française Limitée, 1931, 327 p. $24 \mathrm{~cm}$.

Notre Maître le passé (2e série). Montréal, Granger, 1936. 309 p. $19 \mathrm{~cm}$.

Hocedez, P. Edgar. Histoire de la théologie catholique au XIXe siècle. 3 vol. Vol. II : Epanouissement de la théologie (1831-1870) ; Vol. III: Le règne de Léon XIII (1878-1903). Bruxelles, L'Edition universelle, S.A., Desclée de Bouwer, $1952,1947,418$ p. $23.5 \mathrm{~cm}$. Bibliographie, Index.

Koyré, Alexandre. Etudes d'histoire de la pensée philosophique. Paris, Colin, 1961. 329 p. $23.5 \mathrm{~cm}$.

Lessard, Claude. L'histoire de l'éducation au Collège de Nicolet (1803-1863). Thèse de diplôme d'études supérieures, Facultés des Lettres, Université Laval, 1963.

Maurault, Olivier. Le Petit Séminaire de Montréal. Montréal, Derome, 1918. 240 p. $23.5 \mathrm{~cm}$.

Marges d'histoire. L'art au Canada. Montréal, Librairie d'Action canadienne française Limitée, $1929.310 \mathrm{p} .18 \mathrm{~cm}$.

Paquet, Etienne Théodore. Fragments de l'histoire religieuse et civile de la paroisse de Saint-Nicolas. Lévis, Mercier et Cie, 1894. 400 p. $18.5 \mathrm{~cm}$.

Perrier, Joseph Louis, The Revival of Scholastic Philosophy in the Nineteenth Century. New-York. The Columbia University Press, 1909. 344 p. $22.5 \mathrm{~cm}$. Bibliographie. Index.

Provost, Abbé Honorius. Historique de la Faculté des Arts de l'Université Laval 1852-1952. Québec, publié par la revue 
l'Enseignement secondaire au Canada, 1952, 103 p. $24 \mathrm{~cm}$. Appendices.

Sicard, Abbé Augustin. Les études classiques avant la Révolution. Paris, Didier, Perrin et Cie, 1887. IX-590 p. $19 \mathrm{~cm}$.

Taft Manning, Helen. The Revolt of French Canada 18001835. A Chapter in the History of the British Commonwealth. Londres, MacMillan, 1962 . XX-426 p. $22.5 \mathrm{~cm}$. Index.

Traquair, Ramsay. The Old Architecture of French Canada: $A$ study of the buildings erected in New France from the earliest explorers to the middle of the nineteenth century. Toronto, MacMillan, 1947. XIX - 324 p. $30 \mathrm{~cm}$. Index.

Trudel, Marcel. L'influence de Voltaire au Canada. 2 vol. Montréal, Fides, 1945. 221, 315 p. 20.5 cm. Bibliographie, Index.

Weill, Georges. Histoire de l'enseignement secondaire en France 1802-1920. Paris, Payot, 1920. 255 p. 18.5 cm. Bibliographie.

\section{PÉRIODIQUES}

Beauregard, abbé Lucien. La part de M. Isaac Stanislas Désaulniers à l'introduction du thomisme au Canada français vers l'époque de la renaissance religieuse de 1840 à 1855, in Rapport de la Soc. Can. Hist. Egl. (1941-42) : 77-88.

Drolet, Antonio. La bibliothèque du Séminaire de Québec et son catalogue de 1782, in Le Canada français, XXVIII (1940) : 261-266. La bibliothèque du collège des Jésuites, in RHAF, XIV, 4 (mars 1961) : 487-545.

Gosselin, abbé Auguste. L'abbé Holmes et l'instruction publique, in MSRC (troisième série) 1 (1907-1908) : 127-172.

Hamelin, Louis-Edmond. Evolution numérique séculaire du clergé dans le Québec, in Recherches sociographiques, II, 2 (avril-juin 1961) : 129-241.

Maheux, Mgr Arthur. Pierre Stanislas Bédard 1763-1829: philosophe et savant, in MSRC (troisième série) (1956): 85-93.

Mignon, A. La philosophie cartésienne dans le clergé de France aux débuts du XIXe siècle, in Revue du clergé français, XX (1899), 133-157. La réaction contre le cartésianisme dans le clergé de France aux débuts du XIXe siècle, in Annales de philosophie chrétienne (nouvelle série) XLII (1900) : 397-419. 
Ouellet, Fernand. Mgr Plessis et la naissance d'une bourgeoisie canadienne (1797-1810), in Rapport de la Soc. can. Hist. Egl. (1955-56) : 83-99.

Paquet, Mgr Louis-Adolphe. Coup d'œil sur l'histoire de l'enseignement de la philosophie traditionnelle au Canada, in MSRC (troisième série) XI (1917) : 37-60.

Savard, Pierre. Les débuts de l'enseignement de l'histoire et de la géographie au Petit Séminaire de Québec (1765-1880) in RHAF, XV, 4 (mars 1962) : 509-525; XVI, 1 (juin 1962) : 43-62; XVI, 2 (septembre 1962) : 188-212.

MARC LEBEL 